\title{
Comparative effect of methionine and cysteine supplementation on growth performance and blood profile of Desi chicks
}

\author{
Mehtab Haseena ${ }^{1 *}$, Razia Iqbal ${ }^{1}$, Muhammad Faheem Malik ${ }^{1}$, Syed \\ Usman Haider ${ }^{2}$, Abid Hussain ${ }^{2}$, Tahir Aziz ${ }^{2}$ and Naveed Iftikhar ${ }^{2}$ \\ 1. Department of Zoology, University of Gujrat, Punjab-Pakistan \\ 2. Directorate of Poultry Research Institute Rawalpindi-Pakistan \\ *Corresponding author's email: mehtabhaseena5@gmail.com
}

Citation

Mehtab Haseena, Razia Iqbal, Muhammad Faheem Malik, Syed Usman Haider, Abid Hussain, Tahir Aziz and Naveed Iftikhar. Comparative effect of methionine and cysteine supplementation on growth performance and blood profile of Desi chicks. Pure and Applied Biology. Vol. 8, Issue 4, pp2362-2372.

http://dx.doi.org/10.19045/bspab.2019.80182

Received: 24/05/2019 Revised: 25/07/2019

Accepted: 05/08/2019

Online First: 22/08/2019

\section{Abstract}

Methionine and cysteine are sulfur containing amino acids and have important role in protein synthesis and increases feed efficiency. This study was conducted to evaluate the comparative effect of methionine and cysteine on 22 day old Rhode Island Red (RIR) chicks. The effect of methionine and cysteine was assessed on blood profile and Feed Conversion Ratio (FCR) of Rhode Island Red chicks. Chicks were divided into 7 groups, A,B,C groups were given methionine, having concentrations 0.4 gram per $\mathrm{kg}$ $(\mathrm{g} / \mathrm{kg})$ body weight $(\mathrm{BW}), 0.6 \mathrm{~g} / \mathrm{kg} \mathrm{BW}$ and $0.7 \mathrm{~g} / \mathrm{kg} \mathrm{BW}$ respectively, while groups D,E,F were given the cysteine, having concentrations $0.4 \mathrm{~g} / \mathrm{kg} \mathrm{BW}, 0.6 \mathrm{~g} / \mathrm{kg} \mathrm{BW}$ and $0.7 \mathrm{~g} / \mathrm{kg} \mathrm{BW}$ respectively and $\mathrm{G}$ was control group that was given the normal basal diet. FCR of the chicks was decreased significantly which indicates better growth performance at all concentrations of methionine and cysteine. Hematological parameters including Hemoglobin (Hb), Hematocrit (PCV), Red Blood Cells (RBCs), Mean Corpuscular Hemoglobin (MCH), Mean Corpuscular Hemoglobin Concentration (MCHC) and Mean Corpuscular Volume (MCV) were increased in all treatment groups. It is concluded that sulfur containing amino acid are growth enhancer. These amino acid supplements should be added in animal's diet to improve growth and blood parameters. The study asserts further research on amino acid supplement to examine its effectiveness in other animals.

Keywords: Amino acid supplements; FCR; Hematological parameters; Rhode Island Red Introduction

Two sulfur containing amino acids, Poultry production is increasing day by day. Chicks are taking protein rich diet and are showing better growth. During different growing phases, varying diets are given to the poultry [1]. At the age of four months, desi chicks provide the $0.79 \mathrm{~kg}$ of meat and lay 30 eggs per year [2]. methionine and cysteine are essential for life. These amino acids stabilize protein tertiary structure due to presence of disulfide bond. Major source of cysteine is structural protein such as collagen or keratin which is found in feathers, hair, skin and nails. Highest ratio of methionine is found in egg 
albumin which belongs to protein class globulins. Due to this reason, methionine has become essential element for poultry [3].

Methionine is considered as a first essential amino acid while cysteine is semi essential amino acid in poultry nutrition. Supplements of methionine are required for protein synthesis and increases feed efficiency [4]. Methionine is not produced by chickens so they get methionine through their diets [5]. Dexter laevus (DL)-methionine and DL-2hydroxy-4-methyl butanoic acid (HMBA) are considered as a source of methionine. Liquid DL-HMBA has $88 \%$ purity while powdered DLM has $98 \%$ purity. These two sources have biochemical and physical differences [6].

Methionine serves as a methyl group donor which is involved in non-proteingenic functions. Methyl group is added in the DNA and involved in cell development and differentiation during DNA methylation process [7]. In skeletal muscles, methylation process is affected by methionine present in diet [8]. Methionine can reduce the symptoms for atherosclerosis [9]. Methionine in basal diet increases feed efficiency and egg production. It is also involved in prevention from increased deposition of fats [10]. Oxidative status is related with feed efficiency and lower feed efficiency shows high oxidative status in liver, intestine and breast muscle $[\mathbf{1 1}, \mathbf{1 2}]$.

For best economic results, synthetic methionine is added into the diet. $0.5 \%$ methionine is essential for optimum growth but higher concentrations are required to modulate the immune response of host [13]. In chicks, during the first phase of growth, glutathione peroxidase activity and glutathione redox system is increased by giving higher concentration of methionine [14]. Deficiency in methionine results in reduction of feed efficiency and weight gain [15]. Methionine deficient diet can weaken immune function in broilers by pathological changes in thymus. In the first feeding month of broiler, diet without methionine content results in damaging feed conversion [16]. An increase in apoptotic cells lead to reduction in thymus (T) cell proliferation and decreased $\mathrm{T}$ cell populations [17].

Methionine indirectly acts as precursor of sulphur source and it can be converted irreversibly to cysteine. Naturally, cysteine is derived from duck feathers or human hair. Cysteine has a thiol group and it is involved in formation of disulfide bonds as it undergoes substitution and addition reactions. Cysteine plays vital role in manufacturing antioxidant glutathione, as it involves in electron transfer reaction [18]. Supplementation of cysteine in diet reduces the plasma homocysteine concentration [19]. These two amino acids are part of proteins and involved in regulation of body's homeostasis and performance $[20,21]$. These amino acids affect blood concentrations of heterophile, lymphocytes and stimulate defense function of gastrointestinal tract in chicks $[16,22]$. Methionine and cysteine supplements can alleviate oxidative stress in tissues of chicken [23]. Cysteine synthesizes reduced glutathione which plays role in maintaining counteracts oxidative stress and redox balance in cells [24]. Catalase activity is affected by methionine and it is important in protection of tissues from toxic affect of hydrogen peroxide $\left(\mathrm{H}_{2} \mathrm{O}_{2}\right)$ [25]. Methionine and cysteine are important for maximum growth of commercial broilers [1].

High methionine and cysteine concentration is damaging to growth and immune response of chicks because they contain highly toxic substances including homocysteine and sulfuric acid [26, 27]. No significant relationship has found between cysteine and methionine [19]. Chicks cannot fulfill the requirements of amino acid by the imbalances of amino acid and positive result 
comes from the balances of amino acid in feed [28].

Protein rich diet has less effect on growth performance in growing age of chicks. Cost of desi chicks rearing can be reduced by giving low energy diets in growing age [29]. Lethal dose (LD50) of sulphur containing amino acid is above 2.5 for chicks and half of the chicks showed mortality [30].

Poultry nutritionists considered National Research Council (NRC) as a special guideline for requirement of amino acid for poultry. NRC recommendation considered as a safe estimate for chicks [31]. Levels for methionine and cysteine should be more than the NRC recommended levels [32].

\section{Materials and methods}

\section{Research animals}

This study was carried out on 22 day old 105 desi chicks Rhode Island Red. These chicks were maintained at Govt. poultry farm Sook Kalan Jalal pur jattan road, Gujrat. The 7 groups were made according to different dose concentrations. The groups were A, B, C, D, E, F and G. Replicates such as A1, A2 and A3 were made in each group. Each replicate was carrying 5 birds.

\section{Care and maintenance}

Birds were placed in iron cages. Cages were divided into many parts by partitions. Each part contained three partitions for one group. Optimum temperature was provided to the birds and sheds were made to minimize the heat stress. Regular feed was given to the birds and vaccinated after every week.

\section{Dosage design (Oral)}

Amino acids were obtained from Merck Company. Powdered amino acids mixed in the feed were given to chicks with different time intervals. Group A, B and $\mathrm{C}$ were given methionine with concentrations $(0.4 \mathrm{~g} / \mathrm{kg}$ $\mathrm{BW}, \quad 0.6 \mathrm{~g} / \mathrm{kg} \quad \mathrm{BW}$ and $0.7 \mathrm{~g} / \mathrm{kg} \quad \mathrm{BW}$ ) respectively. Group D, E and F were given cysteine having different concentrations $(0.4 \mathrm{~g} / \mathrm{kg} \mathrm{BW}, 0.6 \mathrm{~g} / \mathrm{kg} \mathrm{BW}$ and $0.7 \mathrm{~g} / \mathrm{kg} \mathrm{BW})$ respectively while group $\mathrm{G}$ was considered as a control group.

\section{Treatment duration}

All treatments including methionine and cysteine had been given from the age of 22 day old chicks and continued for the period of 4 weeks. Three samples were made within the interval of 10 days.

\section{Growth performance}

At the start of experiment, weights of chicks were recorded at $10^{\text {th }}, 20^{\text {th }}$ and $30^{\text {th }}$ days. Before recording the weight, feed was not given to the chicks for 12 hours. Weight of each group was measured separately. Feed conversion ratio was measured as feed intake divided by weight gain $[15,33]$.

$\mathrm{FCR}=$ Total feed intake/total weight gain

\section{Collection of blood sample}

By using vein puncture technique, blood sample was collected. Vacuum sampling tubes having anticoagulant EDTA.K3 was used for blood collection. Wing was pulled and vein was clearly shown between the muscles of biceps and triceps. Bifurcates $\mathrm{v}$ was visible between the wing's vein. Feathers were plucked for the visibility of vein. $70 \%$ alcohol was used to disinfect the area near the bleeding site. Syringe was injected in to the tendon and blood was flowed in to the syringe. This blood was shifted into vacuum sampling tubes having anticoagulant EDTA and by well shaking of tubes, clotting was prevented. These tubes were kept at $37^{\circ} \mathrm{C}$ for preservation [34].

\section{Hematological studies}

After collection of blood, within 24 hours, Automated Haematology Analyzer (model HKTE0112 Guangzhoun Hekang) was used [35]. Coulter method was used for hematological analysis [36].

\section{Statistical analysis}

The SPSS (Statistical Package for Social Science) version 21.0 was used for statistical analysis. The standard error means $( \pm$ S.E.M) for $\mathrm{CBC}$ and FCR were calculated by using Excel. One-way ANOVA was used for data 
analysis. To check variations within these groups, post hoc test was also applied.

\section{Results}

\section{Growth performance}

In (Figure $1 \& 2$ ) it is showing better growth performance as FCR has decreased in RIR chicks after treating them with different doses of methionine $(0.4 \mathrm{~g} / \mathrm{kg}, 0.6 \mathrm{~g} / \mathrm{kg}$ and $0.7 \mathrm{~g} / \mathrm{kg})$ and cysteine $(0.4 \mathrm{~g} / \mathrm{kg}, 0.6 \mathrm{~g} / \mathrm{kg}$ and $0.7 \mathrm{~g} / \mathrm{kg}$ ) respectively.

Moreover, the statistical data analysis for methionine also depicts that mean values for treated group $\mathrm{T} 1 \quad(3.32 \pm 0.008,3.33 \pm 0.005$, $3.20 \pm 0.005) \mathrm{T} 2 \quad(3.23 \pm 0.005,2.55 \pm 0.008$, $2.42 \pm 0.005)$ and T3 $(2.67 \pm 0.008$, $1.77 \pm 0.005,1.64 \pm 0.005)$ are comparatively lower than control group T0 $(3.52 \pm 0.008$, $3.47 \pm 0.008,3.27 \pm 0.005)$. However, FCR is highly decreased in methionine $(0.7 \mathrm{~g} / \mathrm{kg})$ group at $30^{\text {th }}$ day (Figure 1).

Mean values for cysteine for treated group T1 (3.27 $\pm 0.008,3.31 \pm 0.005,3.20 \pm 0.005)$ T2 $(2.85 \pm 0.005,2.67 \pm 0.008,2.44 \pm 0.005)$ and T3 $(2.21 \pm 0.008, \quad 2.16 \pm 0.005$, $2.07 \pm 0.005)$ were significantly decreased with respect to control T0 $(3.52 \pm 0.008$, $3.47 \pm 0.008,3.27 \pm 0.005$ (Figure 2).

\section{Hematological parameters for methionine}

In (Table 1) it is indicating the result for hematological parameters for methionine For WBC at day $10^{\text {th }} \mathrm{T} 1(8.68 \pm 0.09)$, T2 $(8.11 \pm 0.48)$ and T3 $(7.25 \pm 0.46)$ were significantly decreased with respect to control T0 (9.21 \pm 0.29$)$. At $20^{\text {th }}$ and $30^{\text {th }}$ day T1 $(8.25 \pm 0.01,8.21 \pm 0.00)$ has no significant difference with respect to control T0 $(9.33 \pm 0.29,9.43 \pm 0.29)$ but T2 $(6.78 \pm 0.06$, $7.54 \pm 0.00)$ and $\mathrm{T} 3(6.22 \pm 0.01,7.29 \pm 0.01)$ were significantly decreased. However, overall WBCs were decreased by giving methionine in diet.

The RBCs counts at day 10th, 20th and 30th have no significant difference with respect to control except T3. T1 $(4.45 \pm 0.078$, $4.46 \pm 0.01,4.97 \pm 0.01)$ and $\mathrm{T} 2(4.97 \pm 0.01$, $4.99 \pm 0.01,4.99 \pm 0.01)$ showed no significant difference with respect to control T0 $(4.39 \pm 0.145,4.42 \pm 0.145,4.55 \pm 0.145)$ but T3 (5.01 $\pm 0.264,5.18 \pm 0.02,5.01 \pm 0.01)$ were significantly increased. RBCs were increased by increasing concentration of methionine. For $\mathrm{Hb}$ at day $10^{\text {th }}, 20^{\text {th }}$ and $30^{\text {th }}$ T1 (11.91 $\pm 0.14,11.95 \pm 0.02,11.99 \pm 0.00)$ has no significant difference while others T2 $(12.96 \pm 0.577,12.99 \pm 0.01,12.56 \pm 0.02)$ and T3 $(12.37 \pm 0.360,12.99 \pm 0.01,13.01 \pm 0.00)$ were significantly increased with respect to control T0 $(11.82 \pm 0.074, \quad 11.86 \pm 0.074$, $11.90 \pm 0.074)$. Hb levels were improved by giving higher concentration of methionine. HCT levels at $10^{\text {th }}, 20^{\text {th }}$ and $30^{\text {th }} \mathrm{T} 1$ $(37.02 \pm 0.296, \quad 37.73 \pm 0.01, \quad 37.11 \pm 0.01)$ showed no significant difference as compared to control T0 $(36.21 \pm 0.577$, $36.55 \pm 0.577, \quad 36.99 \pm 0.577)$ while T2 $(39.66 \pm 0.881,38.04 \pm 0.02,39.21 \pm 0.01)$ and T3 (40.22 $\pm 1.65,39.56 \pm 0.01,39.99 \pm 0.01)$ were significantly increased. HCT levels were increased by increasing concentration of methionine.

For MCV at day $10^{\text {th }}, 20^{\text {th }}$ and $30^{\text {th }} \mathrm{T} 1$ $(84.77 \pm 0.52, \quad 85.78 \pm 0.01, \quad 85.91 \pm 0.01) \quad \mathrm{T} 2$ $(87.23 \pm 0.43,87.38 \pm 0.23,85.01 \pm 0.00)$ and T3 $(88.90 \pm 0.881,89.99 \pm 0.14,89.99 \pm 0.14)$ were significantly increased as compared to control T0 $\quad(81.49 \pm 0.34, \quad 82.49 \pm 0.34$, $82.99 \pm 0.34)$.

For $\mathrm{MCH}$ at day $10^{\text {th }}, 20^{\text {th }}$ and $30^{\text {th }} \mathrm{T} 1$ $(28.96 \pm 0.31,29.61 \pm 0.01,28.81 \pm 0.03)$ and T2 (29.56 $\pm 0.32, \quad 29.99 \pm 0.02, \quad 29.88 \pm 0.02)$ had no significant difference with respect to control T0 $(28.00 \pm 0.11, \quad 28.45 \pm 0.11$, $28.09 \pm 0.11) \mathrm{T} 3$ at $10^{\text {th }}(29.99 \pm 0.28)$ showed no increase while at $20^{\text {th }}$ and $30^{\text {th }}$ day $(31.00 \pm 0.02, \quad 31.21 \pm 0.02) \quad$ significant increase was observed.

For MCHC at day $10^{\text {th }}, 20^{\text {th }}$ and $30^{\text {th }} \mathrm{T} 1$ $(30.35 \pm 0.27,29.92 \pm 0.03,29.88 \pm 0.01)$ T2 $(30.66 \pm 0.21,30.81 \pm 0.00,30.31 \pm 0.01)$ and T3 (31.29 $\pm 0.26, \quad 30.78 \pm 0.01,30.35 \pm 0.01)$ had no significant difference with respect to 
control T0 $\quad(29.81 \pm 0.22, \quad 29.88 \pm 0.22$, $28.87 \pm 0.22$ ).

For platelets at day $10^{\text {th }}, 20^{\text {th }}$ and $30^{\text {th }} \mathrm{T} 1$ $(187.66 \pm 2.61, \quad 178.00 \pm 1.15, \quad 178.00 \pm 1.15)$ T2 (177.22 $\pm 3.83, \quad 145.33 \pm 0.88$, $166.66 \pm 1.45)$ and T3 (150.33 \pm 3.91 , $155.00 \pm 1.15, \quad 159.33 \pm 1.76) \quad$ were significantly decreased with respect to control T0 $\quad(200.00 \pm 1.59, \quad 205.00 \pm 1.59$, 207.00 \pm 1.59 ).

\section{Hematological parameters for cysteine}

In (Table 2) it is showing the result for hematological parameters for cysteine.

For WBC at day $10^{\text {th }}, 20^{\text {th }}$ and $30^{\text {th }} \mathrm{T} 1$ $(8.34 \pm 0.06, \quad 8.90 \pm 0.01, \quad 8.89 \pm 0.60) \quad$ T2 $(8.99 \pm 0.57,8.24 \pm 0.09,7.98 \pm 0.06)$ and $\mathrm{T} 3$ $(7.88 \pm 0.28, \quad 7.78 \pm 0.06, \quad 7.92 \pm 0.08) \quad$ were significantly decreased with respect to control T0 $\quad(9.21 \pm 0.29, \quad 9.33 \pm 0.29$, $9.43 \pm 0.29)$.

The RBCs counts at day 10th, 20th and 30th T1 $(4.55 \pm 0.08,4.48 \pm 0.09,4.97 \pm 0.01)$ T2 (4.99 $\pm 0.27,4.77 \pm 0.07,4.99 \pm 0.06)$ showed no significant difference while T3 $(4.79 \pm 0.78, \quad 4.98 \pm 0.02, \quad 5.01 \pm 0.06) \quad$ were significantly increased. However, increase in RBCs counts was observed with respect to control T0 $\quad(4.39 \pm 0.145, \quad 4.42 \pm 0.145$, $4.55 \pm 0.145)$.

For $\mathrm{Hb}$ at day $10^{\text {th }} \mathrm{T} 1 \quad(11.87 \pm 0.12) \mathrm{T} 2$ $(11.99 \pm 0.98)$ showed no significant difference with respect to control T0 $(11.82 \pm 0.074, \quad 11.86 \pm 0.074, \quad 11.90 \pm 0.074)$ but T3 $(12.45 \pm 0.28)$ was increased. At $20^{\text {th }}$ and $30^{\text {th }}$ day $\mathrm{T} 1 \quad(12.89 \pm 0.03,12.25 \pm 0.07)$ T2 $(12.98 \pm 0.06, \quad 12.86 \pm 0.06)$ and T3 $(13.01 \pm 0.08,13.01 \pm 0.05)$ were significantly increased. $\mathrm{Hb}$ levels were increased by giving increasing concentration of cysteine.
HCT levels at day $10^{\text {th }}, 20^{\text {th }}$ and $30^{\text {th }} \mathrm{T} 1$ $(37.08 \pm 0.45,37.87 \pm 0.01,37.88 \pm 0.05)$ had no increase as compared to control T0 $(36.21 \pm 0.577, \quad 36.55 \pm 0.577, \quad 36.99 \pm 0.577)$ while T2 $\quad(39.89 \pm 0.10, \quad 38.98 \pm 0.06$, $39.31 \pm 0.08)$ and T3 (39.09 \pm 1.45 , $39.87 \pm 0.06,39.89 \pm 0.06)$ showed significant increase. Overall HCT levels were increased.

For MCV at day $10^{\text {th }}, 20^{\text {th }}$ and $30^{\text {th }} \mathrm{T} 1$ $(82.87 \pm 0.45, \quad 82.88 \pm 0.05, \quad 82.89 \pm 0.01)$ showed no difference with respect to control T0 $\quad(81.49 \pm 0.34, \quad 82.49 \pm 0.34, \quad 82.99 \pm 0.34)$ but T2 $\quad(84.87 \pm 0.98, \quad 85.68 \pm 0.88$, $84.01 \pm 0.06)$ and T3 $(87.09 \pm 0.36$, $88.21 \pm 0.05,88.90 \pm 0.15)$ were significantly increased by increasing concentration of cysteine.

$\mathrm{MCH}$ at $10^{\text {th }}, 20^{\text {th }}$ and $30^{\text {th }}$ days $\mathrm{T} 1$ $(28.78 \pm 0.27, \quad 29.76 \pm 0.06,28.81 \pm 0.03) \quad \mathrm{T} 2$ $(29.98 \pm 0.06,29.88 \pm 0.02,29.87 \pm 0.02)$ and T3 $(29.56 \pm 0.93,30.89 \pm 0.05,30.21 \pm 0.06)$ showed slightly increase as compared to control T0 $\quad(28.00 \pm 0.11, \quad 28.45 \pm 0.11$, $28.09 \pm 0.11$ ).

For MCHC at 10 th, $20^{\text {th }}$ and $30^{\text {th }}$ days $\mathrm{T} 1$ $(30.87 \pm 0.87,29.99 \pm 0.03,29.85 \pm 0.08) \quad \mathrm{T} 2$ $(30.89 \pm 0.27,30.78 \pm 0.09,30.30 \pm 0.04)$ and T3 $(30.78 \pm 0.93,30.65 \pm 0.04, \quad 30.78 \pm 0.06)$ showed no significant difference as compared to control T0 (29.81 \pm 0.22 , $29.88 \pm 0.22,28.87 \pm 0.22$ ).

For Platelets at day $10^{\text {th }}, 20^{\text {th }}$ and $30^{\text {th }} \mathrm{T} 1$ $(194.00 \pm 0.67, \quad 167.00 \pm 1.15, \quad 189.00 \pm 1.15)$ T2 $\quad(178.22 \pm 0.78, \quad 155.33 \pm 0.80$, $176.66 \pm 1.67)$ and T3 $(166.33 \pm 0.45$, $134.00 \pm 1.67, \quad 150.34 \pm 1.98) \quad$ were significantly decreased with respect to control T0 $\quad(200.00 \pm 1.59, \quad 205.00 \pm 1.59$, $207.00 \pm 1.59)$. 
Haseena et al.

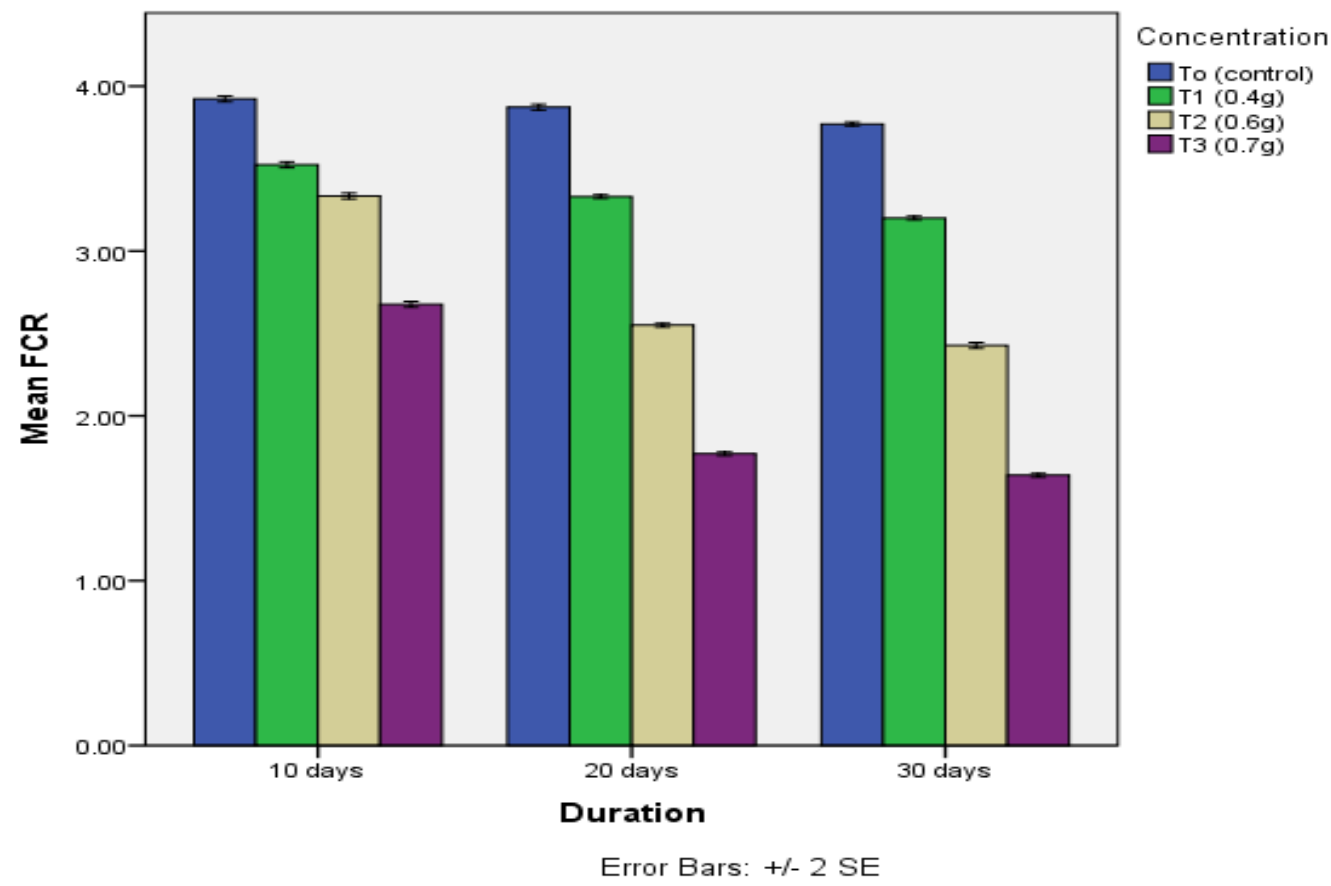

Figure 1. Concentration and duration dependent effect of methionine on feed conversion ratio (FCR) of RIR chicks at $10^{\text {th }}, 20^{\text {th }}$ and $30^{\text {th }}$ day of exposure

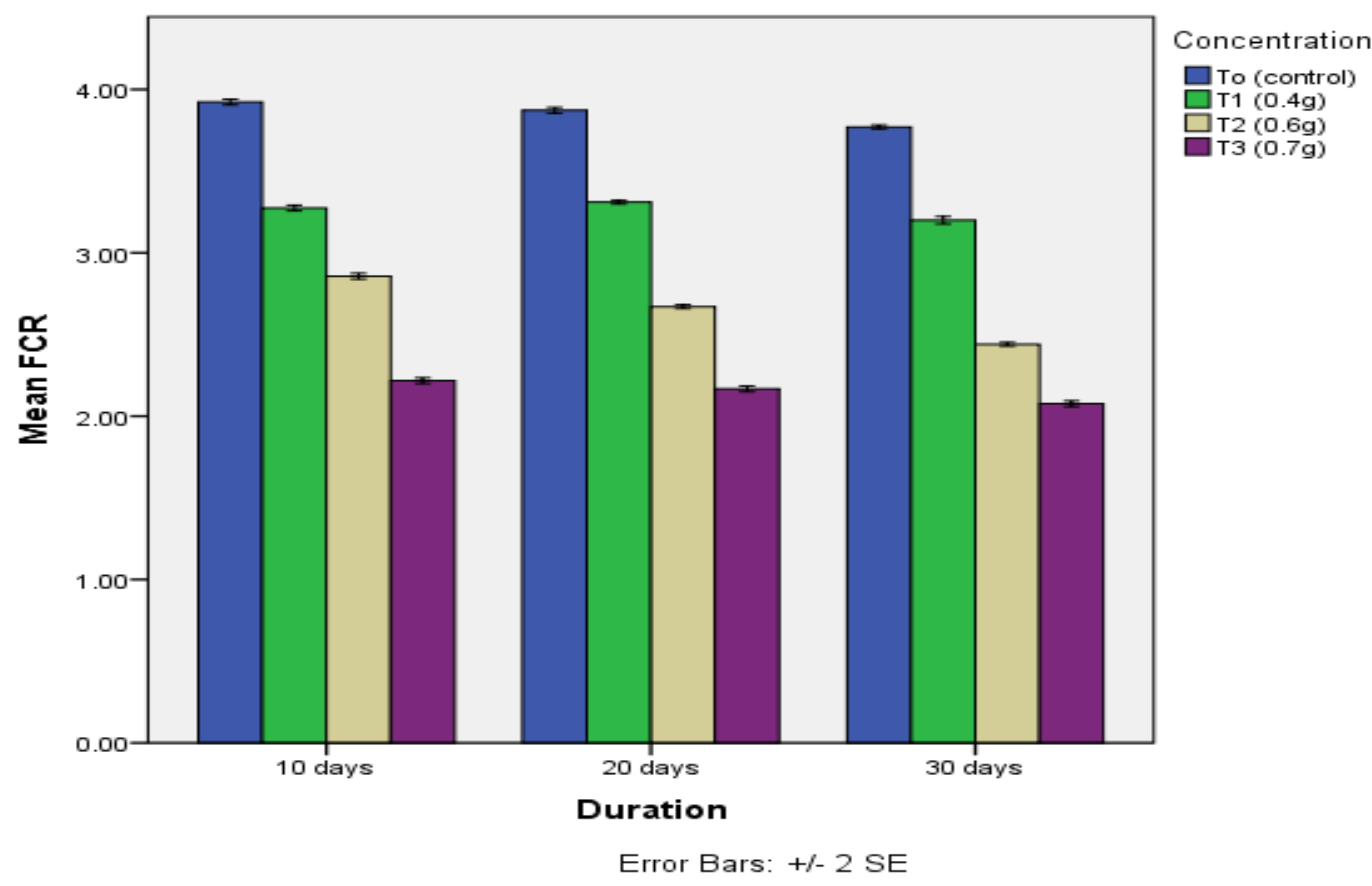

Figure 2. Concentration and duration dependent effect of cysteine on feed conversion ratio (FCR) of RIR chicks at $10^{\text {th }}, 2^{\text {th }}$ and $30^{\text {th }}$ day of exposure 
Table 1. Effect of different dosage of methionine on blood parameters in RIR chicks at 10th, 20th and 30th days (mean \pm S.E.M)

\begin{tabular}{|c|c|c|c|c|c|}
\hline \multirow[b]{2}{*}{ Parameters } & \multirow[b]{2}{*}{ Duration } & \multicolumn{4}{|c|}{ Concentrations Used } \\
\hline & & $\begin{array}{c}\text { T0 } \\
\text { Control } \\
\end{array}$ & $\begin{array}{c}\text { T1 } \\
0.4 \mathrm{~g} / \mathrm{kg} \mathrm{BW}\end{array}$ & $\begin{array}{c}T 2 \\
0.6 \mathrm{~g} / \mathrm{kg} \mathrm{BW} \\
\end{array}$ & $\begin{array}{c}\text { T3 } \\
0.7 \mathrm{~g} / \mathrm{kg} \mathrm{BW}\end{array}$ \\
\hline $\begin{array}{c}\text { WBCs } \\
(\mathrm{x} 103 / \mu \mathrm{l})\end{array}$ & \multirow{8}{*}{ 10th Day } & $9.21 \pm 0.29^{\mathrm{a}}$ & $8.68 \pm 0.09^{b}$ & $8.11 \pm 0.48^{c}$ & $7.25 \pm 0.46^{\mathrm{c}}$ \\
\hline $\begin{array}{c}\text { RBCs } \\
(\mathrm{x} 103 / \mu \mathrm{l})\end{array}$ & & $4.39 \pm 0.145^{\mathrm{a}}$ & $4.45 \pm 0.078^{b}$ & $4.79 \pm 0.296^{c}$ & $5.01 \pm 0.264^{c}$ \\
\hline $\mathrm{Hb}(\mathrm{g} / \mathrm{dL})$ & & $11.82 \pm 0.074^{\mathrm{a}}$ & $11.91 \pm 0.14^{\mathrm{b}}$ & $12.96 \pm 0.577^{b}$ & $12.37 \pm 0.360^{\mathrm{b}}$ \\
\hline $\mathrm{HCT}(\%)$ & & $36.21 \pm 0.577^{\mathrm{a}}$ & $37.02 \pm 0.296^{\mathrm{b}}$ & $39.66 \pm 0.881^{b}$ & $40.22 \pm 1.65^{\mathrm{c}}$ \\
\hline MCV (fL) & & $81.49 \pm 0.34^{\mathrm{a}}$ & $84.77 \pm 0.52^{\mathrm{a}}$ & $87.23 \pm 0.43^{\mathrm{b}}$ & $88.90 \pm 0.881^{\mathrm{b}}$ \\
\hline $\mathrm{MCH}(\mathrm{pg})$ & & $28.00 \pm 0.11^{\mathrm{a}}$ & $28.96 \pm 0.31^{b}$ & $29.56 \pm 0.32^{b}$ & $29.99 \pm 0.28^{c}$ \\
\hline $\mathrm{MCHC}(\mathrm{g} / \mathrm{dL})$ & & $29.81 \pm 0.22^{\mathrm{a}}$ & $30.35 \pm 0.27^{\mathrm{b}}$ & $30.66 \pm 0.21^{\mathrm{b}}$ & $31.29 \pm 0.26^{\mathrm{b}}$ \\
\hline $\begin{array}{l}\text { Platelets } \\
(\mathrm{x} 103 / \mu \mathrm{l}) \\
\end{array}$ & & $200.00 \pm 1.59^{a}$ & $187.66 \pm 2.61^{b}$ & $177.22 \pm 3.83^{\mathrm{b}}$ & $150.33 \pm 3.91^{\mathrm{c}}$ \\
\hline $\begin{array}{c}\text { WBCs } \\
(\mathrm{x} 103 / \mu \mathrm{l})\end{array}$ & \multirow{8}{*}{ 20th Day } & $9.33 \pm 0.29^{\mathrm{a}}$ & $8.25 \pm 0.01^{\mathrm{b}}$ & $6.78 \pm 0.067^{c}$ & $6.22 \pm 0.01^{\mathrm{c}}$ \\
\hline $\begin{array}{c}\text { RBCs } \\
(\mathrm{x} 103 / \mu \mathrm{l})\end{array}$ & & $4.42 \pm 0.145^{\mathrm{a}}$ & $4.46 \pm 0.01^{\mathrm{b}}$ & $4.99 \pm 0.01^{\mathrm{c}}$ & $5.18 \pm 0.02^{c}$ \\
\hline $\mathrm{Hb}(\mathrm{g} / \mathrm{dL})$ & & $11.86 \pm 0.074^{\mathrm{a}}$ & $11.95 \pm 0.02^{\mathrm{b}}$ & $12.99 \pm 0.01^{\mathrm{b}}$ & $12.99 \pm 0.01^{\mathrm{b}}$ \\
\hline $\mathrm{HCT}(\%)$ & & $36.55 \pm 0.577^{\mathrm{a}}$ & $37.73 \pm 0.01^{\mathrm{b}}$ & $38.04 \pm 0.02^{\mathrm{b}}$ & $39.56 \pm 0.01^{\mathrm{c}}$ \\
\hline MCV (fL) & & $82.49 \pm 0.34^{\mathrm{a}}$ & $85.78 \pm 0.01^{\mathrm{a}}$ & $87.38 \pm 0.23^{\mathrm{b}}$ & $89.21 \pm 0.01^{\mathrm{b}}$ \\
\hline $\mathrm{MCH}(\mathrm{pg})$ & & $28.45 \pm 0.11^{\mathrm{a}}$ & $29.61 \pm 0.01^{\mathrm{b}}$ & $29.99 \pm 0.02^{\mathrm{b}}$ & $31.00 \pm 0.02^{\mathrm{c}}$ \\
\hline $\mathrm{MCHC}(\mathrm{g} / \mathrm{dL})$ & & $29.88 \pm 0.22^{\mathrm{a}}$ & $29.92 \pm 0.03^{b}$ & $30.81 \pm 0.00^{\mathrm{b}}$ & $30.78 \pm 0.01^{b}$ \\
\hline Platelets & & $205.00 \pm 1.59^{\mathrm{a}}$ & $178.00 \pm 1.15^{\mathrm{b}}$ & $145.33 \pm 0.88^{b}$ & $155.00 \pm 1.15^{\mathrm{c}}$ \\
\hline $\begin{array}{c}\text { WBCs } \\
(\mathrm{x} 103 / \mu \mathrm{l})\end{array}$ & \multirow{8}{*}{ 30th Day } & $9.43 \pm 0.29^{\mathrm{a}}$ & $8.21 \pm 0.00^{\mathrm{b}}$ & $7.54 \pm 0.00^{c}$ & $7.29 \pm 0.01^{\mathrm{c}}$ \\
\hline $\begin{array}{c}\text { RBCs } \\
(\mathrm{x} 103 / \mu)\end{array}$ & & $4.55 \pm 0.145^{\mathrm{a}}$ & $4.97 \pm 0.01^{\mathrm{b}}$ & $4.99 \pm 0.01^{\mathrm{c}}$ & $5.01 \pm 0.01^{\mathrm{c}}$ \\
\hline $\mathrm{Hb}(\mathrm{g} / \mathrm{dL})$ & & $11.90 \pm 0.074^{\mathrm{a}}$ & $11.99 \pm 0.00^{\mathrm{b}}$ & $12.56 \pm 0.02^{\mathrm{b}}$ & $13.01 \pm 0.00^{\mathrm{b}}$ \\
\hline $\mathrm{HCT}(\%)$ & & $36.99 \pm 0.577^{\mathrm{a}}$ & $37.11 \pm 0.01^{\mathrm{b}}$ & $39.21 \pm 0.01^{\mathrm{b}}$ & $39.99 \pm 0.01^{\mathrm{c}}$ \\
\hline MCV (fL) & & $82.99 \pm 0.34^{\mathrm{a}}$ & $85.91 \pm 0.01^{\mathrm{a}}$ & $85.01 \pm 0.00^{\mathrm{b}}$ & $89.99 \pm 0.14^{b}$ \\
\hline $\mathrm{MCH}(\mathrm{pg})$ & & $28.09 \pm 0.11^{\mathrm{a}}$ & $28.81 \pm 0.03^{b}$ & $29.88 \pm 0.02^{\mathrm{b}}$ & $31.21 \pm 0.02^{\mathrm{c}}$ \\
\hline $\operatorname{MCHC}(\mathrm{g} / \mathrm{dL})$ & & $28.87 \pm 0.22^{\mathrm{a}}$ & $29.88 \pm 0.01^{\mathrm{b}}$ & $30.31 \pm 0.01^{\mathrm{b}}$ & $30.35 \pm 0.01^{\mathrm{b}}$ \\
\hline Platelets & & $207.00 \pm 1.59^{\mathrm{a}}$ & $178.00 \pm 1.15^{\mathrm{b}}$ & $166.66 \pm 1.45^{\mathrm{b}}$ & $159.33 \pm 1.76^{\mathrm{c}}$ \\
\hline
\end{tabular}


Table 2. Effect of different dosage of cysteine on blood parameters in RIR chicks at $10^{\text {th }}$, $20^{\text {th }}$ and $30^{\text {th }}$ days

\begin{tabular}{|c|c|c|c|c|c|}
\hline \multirow[b]{2}{*}{ Parameters } & \multirow[b]{2}{*}{ Duration } & \multicolumn{4}{|c|}{ Concentration Used } \\
\hline & & $\begin{array}{c}\text { T0 } \\
\text { Control }\end{array}$ & $\begin{array}{c}\text { T1 } \\
0.4 \mathrm{~g} / \mathrm{kg} \text { BW }\end{array}$ & $\begin{array}{c}\mathrm{T} 2 \\
0.6 \mathrm{~g} / \mathrm{kg} \mathrm{BW}\end{array}$ & $\begin{array}{c}\text { T3 } \\
0.7 \mathrm{~g} / \mathrm{kg} \mathrm{BW}\end{array}$ \\
\hline WBCs $(\mathrm{x} 103 / \mu \mathrm{l})$ & \multirow{8}{*}{ 10th Day } & $9.21 \pm 0.29^{\mathrm{a}}$ & $8.34 \pm 0.06^{\mathrm{b}}$ & $8.99 \pm 0.57^{\mathrm{b}}$ & $7.88 \pm 0.28^{c}$ \\
\hline RBCs $(x 103 / \mu 1)$ & & $4.39 \pm 0.145^{\mathrm{a}}$ & $4.55 \pm 0.08^{\mathrm{b}}$ & $4.99 \pm 0.27^{\mathrm{c}}$ & $4.79 \pm 0.78^{c}$ \\
\hline $\mathrm{Hb}(\mathrm{g} / \mathrm{dL})$ & & $11.82 \pm 0.074^{\mathrm{a}}$ & $11.87 \pm 0.12^{\mathrm{b}}$ & $11.99 \pm 0.98^{\mathrm{b}}$ & $12.45 \pm 0.28^{\mathrm{b}}$ \\
\hline $\mathrm{HCT}(\%)$ & & $36.21 \pm 0.577^{\mathrm{a}}$ & $37.08 \pm 0.45^{b}$ & $39.89 \pm 0.10^{b}$ & $39.09 \pm 1.45^{\mathrm{c}}$ \\
\hline MCV (fL) & & $81.49 \pm 0.34^{\mathrm{a}}$ & $82.87 \pm 0.45^{\mathrm{a}}$ & $84.87 \pm 0.98^{a}$ & $87.09 \pm 0.36^{\mathrm{a}}$ \\
\hline $\mathrm{MCH}(\mathrm{pg})$ & & $28.00 \pm 0.11^{\mathrm{a}}$ & $28.78 \pm 0.27^{\mathrm{b}}$ & $29.98 \pm 0.06^{\mathrm{b}}$ & $29.56 \pm 0.93^{b}$ \\
\hline $\mathrm{MCHC}(\mathrm{g} / \mathrm{dL})$ & & $29.81 \pm 0.22^{\mathrm{a}}$ & $30.87 \pm 0.87^{b}$ & $30.89 \pm 0.27^{b}$ & $30.78 \pm 0.93^{\mathrm{b}}$ \\
\hline $\begin{array}{l}\text { Platelets } \\
(\mathrm{x} 103 / \mu \mathrm{l})\end{array}$ & & $200.00 \pm 1.59^{\mathrm{s}}$ & $194.00 \pm 0.67^{b}$ & $178.22 \pm 0.78^{b}$ & $166.33 \pm 0.45^{\mathrm{c}}$ \\
\hline WBCs $(x 103 / \mu 1)$ & \multirow{8}{*}{ 20th Day } & $9.33 \pm 0.29^{\mathrm{a}}$ & $8.90 \pm 0.01^{b}$ & $8.24 \pm 0.09^{b}$ & $7.78 \pm 0.06^{\mathrm{c}}$ \\
\hline RBCs $(x 103 / \mu 1)$ & & $4.42 \pm 0.145^{\mathrm{a}}$ & $4.48 \pm 0.09^{b}$ & $4.77 \pm 0.07^{\mathrm{c}}$ & $4.98 \pm 0.02^{\mathrm{c}}$ \\
\hline $\mathrm{Hb}(\mathrm{g} / \mathrm{dL})$ & & $11.86 \pm 0.074^{\mathrm{a}}$ & $12.89 \pm 0.03^{\mathrm{b}}$ & $12.98 \pm 0.06^{\mathrm{b}}$ & $13.01 \pm 0.08^{\mathrm{b}}$ \\
\hline HCT (\%) & & $36.55 \pm 0.577^{\mathrm{a}}$ & $37.87 \pm 0.01^{\mathrm{b}}$ & $38.98 \pm 0.06^{\mathrm{b}}$ & $39.87 \pm 0.06^{\mathrm{c}}$ \\
\hline MCV (fL) & & $82.49 \pm 0.34^{\mathrm{a}}$ & $82.88 \pm 0.05^{\mathrm{a}}$ & $85.68 \pm 0.88^{a}$ & $88.21 \pm 0.05^{\mathrm{a}}$ \\
\hline $\mathrm{MCH}(\mathrm{pg})$ & & $28.56 \pm 0.11^{\mathrm{a}}$ & $29.76 \pm 0.06^{\mathrm{b}}$ & $29.88 \pm 0.02^{\mathrm{b}}$ & $30.89 \pm 0.05^{\mathrm{b}}$ \\
\hline $\mathrm{MCHC}(\mathrm{g} / \mathrm{dL})$ & & $29.88 \pm 0.22^{\mathrm{a}}$ & $29.99 \pm 0.03^{b}$ & $30.78 \pm 0.09^{b}$ & $30.65 \pm 0.04^{b}$ \\
\hline Platelets & & $205.00 \pm 1.59^{\mathrm{a}}$ & $167.00 \pm 1.15^{b}$ & $155.33 \pm 0.80^{b}$ & $134.00 \pm 1.67^{c}$ \\
\hline WBCs $(x 103 / \mu 1)$ & \multirow{8}{*}{ 30th Day } & $9.43 \pm 0.29^{\mathrm{a}}$ & $8.89 \pm 0.60^{\mathrm{b}}$ & $7.98 \pm 0.06^{\mathrm{b}}$ & $7.92 \pm 0.08^{\mathrm{c}}$ \\
\hline RBCs $(x 103 / \mu)$ & & $4.55 \pm 0.145^{\mathrm{a}}$ & $4.97 \pm 0.01^{\mathrm{b}}$ & $4.99 \pm 0.06^{\mathrm{c}}$ & $5.01 \pm 0.06^{\mathrm{c}}$ \\
\hline $\mathrm{Hb}(\mathrm{g} / \mathrm{dL})$ & & $11.90 \pm 0.074^{\mathrm{a}}$ & $12.25 \pm 0.07^{\mathrm{b}}$ & $12.86 \pm 0.06^{\mathrm{b}}$ & $13.01 \pm 0.05^{\mathrm{b}}$ \\
\hline $\mathrm{HCT}(\%)$ & & $36.99 \pm 0.577^{\mathrm{a}}$ & $37.88 \pm 0.05^{\mathrm{b}}$ & $39.31 \pm 0.08^{\mathrm{b}}$ & $39.89 \pm 0.06^{\mathrm{c}}$ \\
\hline MCV (fL) & & $82.99 \pm 0.34^{\mathrm{a}}$ & $82.89 \pm 0.01^{\mathrm{a}}$ & $84.01 \pm 0.06^{\mathrm{a}}$ & $88.90 \pm 0.15^{\mathrm{a}}$ \\
\hline $\mathrm{MCH}(\mathrm{pg})$ & & $28.09 \pm 0.11^{\mathrm{a}}$ & $28.81 \pm 0.03^{\mathrm{b}}$ & $29.87 \pm 0.02^{\mathrm{b}}$ & $30.21 \pm 0.06^{\mathrm{b}}$ \\
\hline $\mathrm{MCHC}(\mathrm{g} / \mathrm{dL})$ & & $28.87 \pm 0.22^{\mathrm{a}}$ & $29.85 \pm 0.08^{b}$ & $30.30 \pm 0.04^{\mathrm{b}}$ & $30.78 \pm 0.06^{\mathrm{b}}$ \\
\hline Platelets & & $207.00 \pm 1.59^{\mathrm{a}}$ & $189.00 \pm 1.15^{\mathrm{b}}$ & $176.66 \pm 1.67^{b}$ & $150.34 \pm 1.98^{c}$ \\
\hline
\end{tabular}

\section{Discussion}

Feed conversion ratio of RIR chicks was reduced significantly which showed positive effect and better growth performance. Experimental group used higher concentrations of methionine and cysteine in comparison to control group. Increase in metabolism may be due to higher uptake of amino acids. Hematological parameters were also affected as $\mathrm{Hb}, \mathrm{PCV}$ and MCV were increased.

According to a study [37] better performance and high breast muscle yield were observed in light weighed chicks as compared to heavy chicks. In another study [38] birds who take high concentrations of methionine showed better performance and it is associated with adequate amount of essential amino acid especially methionine [39]. Methionine mixed with corn-soyabean diet results in good performance of chicks [40].

Treated groups showed decreased FCR while control group has high FCR. At $30^{\text {th }}$ day, methionine having concentration $(0.7 \mathrm{~g} / \mathrm{kg})$ showed highly decreased FCR and this result shows compatibility with [35] who performed an experiment on broiler 
chicks and chicks showed better performance when they were fed on methionine supplement.

Methionine supplement increased the blood parameters. Moreover, methionine is also involved in muscle development, synthesis of others amino acid and digestion of feed stuff [41]. Blood parameters for treated groups were compared with control group. Increases in $\mathrm{Hb}, \mathrm{PCV}$ and $\mathrm{RBCs}$ were observed as compared to control groups [10]. Chicks showed better performance by giving cysteine [15]. Birds fed diets containing $0.35 \%$ cysteine and $0.50 \%$ methionine showed higher body weight gain and high performance as compared to other dietary treatments.

The statistical analysis depicts a decreased FCR which is showing better growth performance. $\mathrm{RBCs}, \mathrm{Hb}$ and $\mathrm{PCV}$ were significantly increased at different concentrations of methionine $(0.4 \mathrm{~g} / \mathrm{kg}$, $0.6 \mathrm{~g} / \mathrm{kg}, \quad 0.7 \mathrm{~g} / \mathrm{kg})$ and cysteine $(0.4 \mathrm{~g} / \mathrm{kg}$, $0.6 \mathrm{~g} / \mathrm{kg}, \quad 0.7 \mathrm{~g} / \mathrm{kg})$. This result was compatible with those reported by [10] who performed an experiment on hematological parameters of chicks. A significant $(\mathrm{P}<0.05)$ increase in $\mathrm{Hb}, \mathrm{PCV}$ and RBCs was observed when compared with control group.

\section{Conclusion}

The study revealed that sulfur containing amino acid including methionine and cysteine have positive role in growth performance. FCR was reduced which indicates better growth performance of birds at all concentration of methionine and cysteine. Hematological parameters such as Red Blood Cells (RBCs), Hemoglobin (Hb), Mean Corpuscular Hemoglobin (MCH), Mean Corpuscular Hemoglobin Concentration (MCHC) and Mean Corpuscular Volume (MCV) were increased for all treatment groups. This study confirms that higher amino acid concentrations are growth enhancer and increases blood parameters.

\section{Author's contributions}

Conceived and designed the experiments: $\mathrm{M}$ Haseena, R Iqbal \& MF Malik, Performed the experiments: $M$ Haseena, Analyzed the data: M Haseena, Contributed materials/ analysis/ tools: SU Haider, A Hussain, T Aziz \& N Iftikhar, Wrote the paper: M Haseena.

\section{References}

1. Farkhoy M, Modirsanei M, Ghavidel O, Sadegh M \& Jafarnejad S (2012). Evaluation of Protein Concentration and Limiting Amino Acids Including Lysine and Met + Cys in Prestarter Diet on Performance of Broilers. Vet Med Int 3: 1-7.

2. Sahota AW \& Bhatti BM (2003). Productive performance of Desi field chickens as affected under deep litter system. Pak Vet J 1(1): 35-38

3. Wilke $\mathrm{T}$ (2014). Methionine Production- a Critical Review. Appl Microbiol Biot 98(24): 9893-9914.

4. Kidd MT (2004). Nutritional modulation of immune function in broilers. Poult Sci 83: 650-657.

5. Saengkerdsub S, O'Bryan CA, Crandall PG \& Ricke1 SC (2013). Possibility for Probiotic Sources of Methionine for Organic Poultry Nutritional Supplementation: An Early Review. J Prob Health 1: 1-7.

6. Sangali CP, Bruno DG, Nunes RV, Neto AR, Pozza PC \& Moraes TM (2014). Bioavailability of different methionine sources for growing broilers. $R$ Bras Zootec 43(3): 140-145.

7. Niculescu MD \& Zeisel SH (2002). Diet, methyl donors and DNA methylation: interactions between dietary folate, methionine and choline. $J$ Nutr 132: 2333-2335.

8. Liu G, Zong K, Zhang L \& Cao S (2010). Dietary methionine affects meat 
quality and myostatin gene exon 1 region methylation in skeletal muscle tissues of broilers. Agri Sci China 9: 1338-1346.

9. Soeken KL, Lee WL, Bausell RB, Agelli M \& Berman BM (2002). Safety and efficacy of S-adenosylmethionine (SAMe) for osteoarthritis. J Fam Pract 51: 425-430.

10. Wahed AA (2016). Effect of feeding lysine and methionine on some production performance and blood picture parameter of broiler chickens. AL-Qadisiya J of Vet Med Sci 15(1): 1821.

11. Bottje WG, Iqbal $M$, Pumford NR, Ojano-Dirain C \& Lassiter K (2004). Role of mitochondria in the phenotypic expression of feed efficiency. $J$ Appl Poultry Res 13: 94-105.

12. Iqbal $\mathrm{M}$, Pumford $\mathrm{NR}$, Tang $\mathrm{ZX}$, Lassiter K, Wing T, Cooper M \& Bottje $\mathrm{W}$ (2004). Low feed efficient broilers within a single genetic line exhibit higher oxidative stress and protein expression in breast muscle with lower mitochondrial complex activity. Poult Sci 83: 474-484.

13. Jankowski J, Kubińska M, Juśkiewicz J, Czech A \& Zduńczyk Z (2016). The effect of dietary methionine levels on fattening performance and selected blood and tissue parameters of turkeys. Arch Anim Nutr 70: 127-140.

14. Nemeth K, Mezes M, Gaal T, Bartos A, Balogh K \& Husveth F (2004). Effect of supplementation with methionine and different fat sources on the glutathione redox system of growing chickens. Acta Vet Hung 52: 369-378.

15. Goulart CC, Perazzo Costa FG, Da Silva JH, De Souza JG \& Rodrigues VP (2011). Requirements of digestible Methionine + cystine for broiler chickens at 1 to 42 days of age. $R$ Bras Zootec 40: 797-803.
16. Bouyeh M (2012). Effect of excess lysine and methionine on immune system and performance of broilers. Ann Biol Res 3: 3218-3224.

17. Wu B, Cui H, Peng X, Fang J, Cui W \& Liu X (2012). Effect of methionine deficiency on the thymus and the subsets and proliferation on peripheral blood T cell, and serum IL 2 in broilers. J Int Agri 11: 1009-1019.

18. Piste P (2013). Cysteine-master antioxidant. Int J Pharm Chem Biol Sci 3(1): 143-149.

19. Xie M, Hou SS, Huang W, Zhao L, Yu JY, \& Li WY (2004). Interrelationship between Methionine and Cystine of Early Peking Ducklings. Poult Sci 83: 1703-1708.

20. Brosnan JT \& Brosnan ME (2006). The sulfur-containing amino acids: an overview. J Nutr 136: 1636-1640.

21. Bunchasak C (2009). Role of dietary methionine in poultry production. $J$ Poult Sci 46: 169-179.

22. Ruth MR \& Field CJ (2013). The immune modifying effects of amino acids on gut-associated lymphoid tissue. J Anim Sci Biotechnol 42: 27

23. Swennen Q, Geraert PA, Mercier Y, Everaert N, Stinckens A \& Willemsen $H$ (2011). Effects of dietary protein content and 2-hydroxy-4methylthiobutanoic acid or DLmethionine supplementation on performance and oxidative status of broiler chickens. Br J Nutr 106: 18451854.

24. Rahman I, Biswas SK, Jimenez LA, Torres M \& Forman HJ (2005). Glutathione, stress responses, and redox signaling in lung inflammation. Antioxid Redox Signal 7: 42-59.

25. Aydemir T \& Kuru K (2003). Purification and Partial Characterization of Catalase from Chicken Erythrocytes and the Effect of Various Inhibitors on 
Enzyme Activity. Turk J Chem 27: 8597.

26. $\mathrm{Wu}$ G \& Meininger CJ (2002). Regulation of nitric oxide synthesis by dietary factors. Аnпи Rev Nutr 22: 6186.

27. Li P, Yin YL, Li D, Kim SW \& Wu G (2007). Amino acids and immune function. Brit J Nutr 98: 237-252.

28. Hadinia S, Shivazad M, Moravej H, Alahyari-shahrasb M, \& Nabi MM (2014). Bio-efficacy comparison of herbal-methionine and DL-methionine based on performance and blood parameters of broiler chickens. Vet Res Forum 5: 81-87.

29. Anjum MS, \& Khan SH (2008). Effects of different energy protein ratio on the performance of desi native chickens during growing phase. Asian J Poultry Sci 2: 42-47.

30. Baker DH (2006). Comparative Species Utilization and Toxicity of Sulfur Amino Acids. J Nutr 136(6): 16701675.

31. Anonymous (1994). National Research Council: Nutrient Requirements of Poultry. The National Academies Press, Washington DC, USA.

32. Sklan D \& Noy Y (2005). Direct Determination of Optimal Amino Acid Intake for Maintenance and Growth in Broilers. Poult Sci 84: 412-418.

33. Al-Dobaib SN (2010). Effect of diets on growth, digestibility, carcass and meat quality characteristics of four rabbit breeds. Saudi J Biol Sci 17: 83-93.

34. Ackerman Z, Oron-Herman M, Grozovski M, Rosenthal T \& Pappo O (2005). Fructose-induced fatty liver disease. Hypertension 45(5):1012-1018.

35. Yodseranee R \& Bunchasak C (2012). Effect of dietary methionine source on productive performance, blood chemical and haematological profiles in broiler chicken under tropical conditions. Trop Anim Health Prod 44: 1957-1963.

36. Ghazy AE, Gomaa NA \& Nasr NE (2016). Hematological and Biochemical Evaluation in Holstein-Friesian Cows Before and After Surgical Correction of Left Abomasal Displacement on-Filed Condition. Alexandria $J$ of Vet $\mathrm{Sci}$ 49(1): 138-146.

37. Wen $\mathrm{C}$, Chen $\mathrm{Y}, \mathrm{Wu}$, Wang $\mathrm{T}$, \& Zhou Y (2014). MSTN, mTOR and FoxO4 are involved in the enhancement of breast muscle growth by metthionine in broilers with lower hatching weight. Plos One 9:e114236.

38. Maroufyan E, Kasim A \& Hashemi SR (2010). Change in growth performance and liver function enzymes of broiler chickens challenged with infectious bursal disease virus to dietary supplementation of methionine and threonine. A J Anim Sci 5(1): 20-26.

39. Swain BK \& Johri TS (2000). Effect of supplemental methionine, choline and their combinations on the performance and immune response of broilers. Br Poult Sci 41: 83-88.

40. Hesabi A, Nasiri H, \& Birjandi M (2006). Effect of supplemental methionine and lysine on performance and carcass yield characteristics in broiler chicks. Proceedings of the 8th Conference on European Poultry, Sep. 10-14, Verona, Italy.

41. Haque ME, Mustari A \& Rahman MM (2014). Study on the effect of enzyme and methionine on growth performance and hemato-biochemical parameters in broilers. J Mater Res 1(1): 1-13. 